\title{
Should clinical complications affect USDA pain categories?
}

"Oh my God, what did I do?" was the frightened cry of Gina Hill, her petite frame quivering with fear, her eyes filling with tears. There, on a table in front of her, was a young female rabbit, almost immobile after being dropped no more than one foot. Hill was an experienced animal care technician and knew that the rabbit was part of an osteoporosis study. The rabbit had given a hard kick, dug its hind nails into Hill's arm and pushed itself free before Hill could do anything. Sobbing, she carefully picked up the rabbit, put it back in its cage and called Steve Margolis, the attending veterinarian.

Margolis was there within minutes and examined the still-frightened animal. "Is it a broken back?" Hill asked. "I don't think so," said Margolis. "It feels more like a tibial fracture, but I'll take an X-ray to be sure." "Can you fix it, Steve?" Hill asked. "Maybe," he replied, "but the injury itself and the problem of trying to repair a fracture in an animal that already has poor bone density may make the rabbit unsuitable for the study." "Well, I'll wait and see," said Hill. "Maybe the investigator will let me adopt the rabbit."

The rabbit did have a fractured tibia, but the bone ends were well aligned. Margolis was able to place a soft cast around the leg, which, surprisingly, the rabbit barely bothered. Hill was allowed to adopt the animal after the cast was removed. When it was time to file the USDA annual report, Margolis asked if the rabbit was to be placed in pain or distress category C, D or E. "Wasn't this a category $\mathrm{C}$ (no pain or distress) study to begin with?" asked the IACUC chairman, Larry Covelli. "Yes," responded Margolis. “Then it's still category C," said Covelli. "I'm not so sure about that," said Margolis. "Here's the problem. The rabbit started out as category C, but it was injured. I used anesthesia to cast the leg, and I gave an analgesic after the accident, so maybe it's category D (alleviated pain or distress). But it took me a few minutes to get to the rabbit and about 30 more minutes before I gave the analgesic, so perhaps it should be in category E (unalleviated pain or distress). What do you think, Larry?" "I think the whole incident was a clinical problem and the USDA category is still category C," answered Covelli.

Margolis left and read the Animal Welfare Act regulations. He couldn't find anything to suggest that a clinical problem that apparently was caused, in part, by the ongoing osteoporosis research required the rabbit to remain in category $C$. In fact, as far as he could tell, it made no difference if a clinical problem was related or unrelated to the research. The pain or distress category, he believed, was dependent on what the animal actually experienced, not the cause of the problem. Covelli disagreed. He believed that clinical problems and their treatment, whether or not they emanated from a research project, were excluded from determining the USDA pain or distress categories. What do you think?

\section{RESPONSE}

\section{Concur with Covelli}

\section{Lisa Portnoy, DVM, DACLAM \& Monica Bur, BS, LATG}

There are two issues for discussion in this scenario: whether the rabbit should remain in its original assigned pain and distress category for the USDA annual report after clinical intervention, and whether it matters if the rabbit's injury was a spontaneous occurrence or a result of the study. Margolis assumed that the injury was caused in part by the osteoporosis study and that this rabbit should therefore be reported in a different pain and distress category because of the subsequent necessary clinical interventions.
There is no specific language in the Animal Welfare Act regulations to indicate whether or not clinical interventions should be considered when assigning animals to pain and distress classifications in the annual report ${ }^{1}$. If all unanticipated injuries, pain and distress were to be accounted for when writing this (or any) protocol, all animals would be placed in category $\mathrm{E}$ for all studies. USDA Policy 11 gives guidance regarding what may be considered a painful or distressful procedure and indicates that animals must be reported in the proper pain category in the final report ${ }^{2}$. USDA Policy 17 addresses pain and distress categories as they apply to teaching, research, experiments or testing: "List all locations where animals were housed or used in actual research, testing, teaching, or experimentation, or held for these purposes"3.
Perhaps the policy that comes closest to specifying that clinical interventions should not be used to classify animals in the annual report is USDA Policy 14, which states, "No animal assigned to a proposal is to be used in more than one major survival operative procedure... However, an animal that has an emergency major operative procedure as part of proper veterinary care may still be used in a proposal that requires a major survival operative procedure"4. Veterinary medical care here (a major surgical procedure) is considered separate from procedures allowed for research purposes; this is consistent with Covelli's beliefs.

In keeping with USDA Policy 14, in order for the rabbit to remain in category $\mathrm{C}$, the tibial fracture should have been spontaneous and not be a direct result of 